\title{
Silencing of EphA2 inhibits invasion of human gastric cancer SGC-7901 cells in vitro and in vivo
}

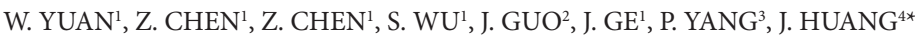 \\ ${ }^{1}$ Department of General Surgery, Xiangya Hospital, Central South University, Xiangya Road, Changsha 410008, Hunan, P. R. China; ${ }^{2}$ Department \\ of General Surgery, Yiyang Central Hospital, Kangfu Road, Yiyang 413000, Hunan, P. R. China; ${ }^{3}$ Hepatobiliary \& Enteric Surgery Research \\ Center, Central South University, Xiangya Road, Changsha 410008, Hunan, P. R. China; ${ }^{4}$ Department of Oncology, Xiangya Hospital, Central \\ South University, Changsha, Hunan, P. R. China
}

*Correspondence: chenzihua1@hotmail.com

Received July 28, 2011 / Accepted August 29, 2011

\begin{abstract}
Receptor tyrosine kinases (RTKs), the common products of transforming oncogenes, have been widely used as indicators in the genesis and progression of human tumors. Until now, the erythropoietin-producing human hepatocellular (Eph) receptors have been recognized as the largest family of RTKs. EphA2, one member of Eph receptors, locates on human chromosome 1 p36.1 which is a hot region for cancer research. It has been reported that high EphA2 expression levels were correlated with the tumor metastasis and poor prognosis. Increased expression of EphA2 can promote tumor growth and enhance the metastatic potential. To further define the function of EphA2 in malignant invasion, we employed the small interference RNA (siRNA) technique to knockdown gene expression of EphA2 in the gastric cancer SGC-7901 cell. Our results showed that the expression of double stranded RNA led to the efficient and specific inhibition of endogenous EphA2 expression in SGC-7901 cells. Silencing of EphA2 expression inhibited cell proliferation, caused cell cycle arrest, and decreased cell invasion in vitro. In addition, intratumoral injection EphA2 siRNA plasmid suppressed the growth of SGC-7901 cells xenografts in nude mice. Furthermore, knockdown of EphA2 expression reduced the expression of matrix metalloproteinase-9 (MMP-9) in vitro and in vivo. In conclusion, our findings demonstrate that silencing of EphA2 inhibits gastric cancer SGC-7901 cell proliferation, invasion and MMP-9 expression, which indicate that the specific inhibition of EphA2 may be a potential approach for gastric cancer therapy.
\end{abstract}

Key words: EphA2, gastric cancer, MMP-9, SGC-7901, siRNA, tumor invasion

Receptor tyrosine kinases (RTKs), the common products of transforming oncogenes, have been documented or suspected to be involved in mediating processes leading to the genesis and progression of malignancy [1]. Until now, the erythropoietin-producing hepatocellular (Eph) receptors have been recognized as the largest family of RTKs, which contains 14 distinct receptors including eight identified ligands. In the Eph family, EphA2 that locates on human chromosome 1p36.1 is expressed at a lowest level in epithelial cells [2]. However, elevated EphA2 was frequently observed in human cancers and associated with tumor progression, including glioblastoma, non-small cell lung cancer, hepatocellular carcinoma, colorectal and endometrial cancer [3-7]. We have previously demonstrated that overexpressed EphA2 was detected in 77.3\% of gastric cancer patients and correlated with the metastasis and poor prognosis [8]. Moreover, EphA2 overexpression pro- moted ovarian cancer growth by enhancing cell-extracellular matrix (ECM) adhesion, increasing anchorage-independent growth [9]. However, the exact mechanisms for EphA2 in gastric cancer invasion are complicated and need further study.

Matrix metalloproteinases (MMPs), a group of zincdependent endopeptidases, have been well documented to have important functions in cancer invasion and metastasis mediated principally by their activities in degrading ECM and basement membrane [10]. Among the MMPs, MMP-9 (gelatinase-B) has been paid much attention because it induces cancer metastasis by degrading denatured collagens (gelatins) and type IV collagen, which are the major structural component of the ECM [11]. Higher expression of MMP-9 is involved in the progression of many cancers, including gastric cancer $[12,13]$. There is considerable evidence to indicate that MMP9 is the most important mediators of tumor migration and 
invasion among MMPs [14]. In our previous study, a positive correlation between EphA2 and MMP-9 expressions has also been confirmed in hepatocellular carcinoma [15]. However, it is undefined that EphA2 receptor may promote invasion of tumor cells via regulation of MMP-9.

In the present study, we first developed an efficient EphA2 siRNA plasmid to specifically silence the expression of EphA2 in gastric cancer cells. Using this plasmid, we examined the effects of suppressing EphA2 expression on cellular proliferation, invasiveness, and MMP-9 expression in vitro. In addition, we evaluated the effect of silencing EphA2 in vivo using a nude mouse xenograft model.

\section{Materials and methods}

Cell culture. The human gastric adenocarcinoma cell line SGC-7901 was purchased from the Xiangya Central Experiment Laboratory, Central South University (Changsha, China). The cells were cultured in PRMI 1640 Media (Hyclone, Waltham, USA) supplemented with $10 \%$ fetal bovine serum (FBS, Hyclone, Waltham, USA), $100 \mathrm{IU} / \mathrm{ml}$ penicillin and $100 \mathrm{mg} / \mathrm{ml}$ streptomycin in a humidified atmosphere of 37 ${ }^{\circ} \mathrm{C}$ at $5 \% \mathrm{CO}_{2}$.

Plasmid construction and transfection. The siRNA sequences used for targeted silencing of EphA2 gene (NCBI Ref Seq NM_004431.2) were previously tested to be efficacious according to Duxbury et al [16]. The sequences of sense and antisense of EphA2 siRNA were: 5'-TGACATGCCGATCTACATG-3' and 5'-CATGTAGATCGGCATGTCA-3'. A non-silencing siRNA was used as a negative control and the sequences of sense were: 5'-TTCTCCGAACGTGTCACGT3 ' and 5'-ACGTGACACGTTCGGAGAA-3', which had no significant homology to human or mouse gene sequences. The pGCsilencerU6-EphA2 siRNA and non-silencing siRNA plasmid (U6-shRNA-CMV-EGFP) containing a CMV-driven GFP reporter, a U6 promoter upstream of the cloning restriction sites (HpaI and XhoI to allow the introduction of EphA2 shRNA or non-silencing shRNA plasmid vector) were constructed by GeneChem Biomedical Co., Ltd (Shanghai, China). A total of $5 \times 10^{5}$ SGC-7901 cells were seeded into each well of a 6-well tissue culture plate. When the cells reached $80-90 \%$ confluence on the day of transfection, the specific EphA2 siRNA or non-silencing siRNA was transfected into the cells using RNAiFect Transfection Reagent (Qiagen, Valencia, USA) according to the manufacturer's protocol. Three groups of cells were used in the in vitro studies, including untransfected cells (black control group), cells transfected with non-silencing siRNA (negative control group) and cells transfected with EphA2 siRNA (EphA2 siRNA group).

Real-time reverse transcriptase polymerase chain reaction (Real-time RT-PCR). $5 \times 10^{5}$ cells from each group were seeded into a 6-well cell culture plate and harvested after 48 $\mathrm{h}$ in culture. Total RNA of cell lines and tissues were extracted using the Trizol reagent (Invitrogen, Carlsbad, USA) following the manufacturer's instructions. The cDNA pool was synthesized using $1 \mu \mathrm{g}$ of total RNA and TaqMan ${ }^{\circledR}$ Reverse Transcription Reagents (Applied Biosystems, Foster City, USA) as described by the manufacturer. The primers for EphA2, MMP-9 and $\beta$-actin (internal control) were as follows: EphA2 primer, forward 5'-ATGGAGCTCCAGGCAGCCCGC-3', reverse 5'-GCCATACGGGTGTGTGAGCCAGC-3'; MMP9 primer, forward 5'- TGGGCTACGTGACCTATGACAT-3', reverse 5'- GCCCAGCCCACCTCCACTCCTC-3'; $\beta$-actin primer, forward 5'- CCTGTACGCCAACACAGTGC-3', reverse 5'- ATACTCCTGCTTGCTGATCC -3'. EphA2, MMP-9 and $\beta$-actin genes were amplified from the cDNA pool using gene specific primers and Power SYBR ${ }^{\circledR}$ Green PCR Master Mix (Applied Biosystems, Foster City, USA) in a ABI PRISM ${ }^{\circ}$ 7700 Sequence Detection System (Applied Biosystems, Foster City, USA). The PCR cycling conditions employed were as follows: $95^{\circ} \mathrm{C} / 5 \mathrm{~min}$, and $95^{\circ} \mathrm{C} / 30 \mathrm{~s}, 60^{\circ} \mathrm{C} / 1 \mathrm{~min}, 72^{\circ} \mathrm{C}$ $/ 1$ min for 40 cycles. Quantitative RT-PCR was done at least thrice, including a no-template control as a negative control. The relative levels of EphA2 and MMP-9 mRNA expression were expressed as the ratio of EphA2 or MMP- 9 to $\beta$-actin and calculated from the standard curve as directed. All reported results are the average ratios of three different independent experiments.

Western blot. For western blot analysis, $5 \times 10^{5}$ cells were seeded in a $60 \mathrm{~mm}$ culture plate, followed by siRNA transfection. At $48 \mathrm{~h}$ post-transfection, the whole cell extracts were prepared using $0.14 \mathrm{M} \mathrm{NaCl}, 0.2 \mathrm{M}$ triethanolamine, $0.2 \%$ sodium deoxycholate, $0.5 \%$ Nonidet P-40 and supplemented with a protease inhibitor (all the products were from Sigma, St. Louis, Missouri, USA). Each $50 \mu \mathrm{g}$ of SDS sample was loaded onto $12 \%$ SDS-PAGE gels and blotted onto an Optitran BA-S85 membrane (Schlischer \& Schell, Dassel, Germany). The transferred membranes were subsequently incubated overnight at $4^{\circ} \mathrm{C}$ with rabbit antibody against EphA2 (dilution 1:1000, sc-924) or goat antibody against MMP-9 (dilution 1:1000, sc6840) (both from Santa Cruz, CA, USA). After washing, they were incubated with horseradish peroxidase (HRP)-linked antibody IgG (Amersham Biosciences, Piscataway, USA) for $1 \mathrm{~h}$ at room temperature. Bands were visualized by employing the ECL AdvanceTM Detection System (Amersham Biosciences, Piscataway, USA). EphA2 or MMP-9 protein expression levels were represented as the densitometric ratio of the targeted protein to $\beta$-actin protein. All reported results were the average ratio gotten from three different independent experiments.

MTT assay. Cell proliferation was assessed by methylthiazolyldiphenyl-tetrazolium bromide (MTT) assay. SGC-7901 cells were incubated in 96-well plates at a /density of $1 \times 10^{4}$ cells per well with PRMI 1640 Media supplemented with 10\% fetal bovine serum for $24 \mathrm{~h}$. After transfection (three groups) for $24,48,72$, and $96 \mathrm{~h}, 10 \mu \mathrm{l}$ of sterile MTT dye $(5 \mathrm{mg} / \mathrm{ml}$, Sigma-Aldrich, St. Louis, USA) was added and incubated for another $4 \mathrm{~h}$ at $37^{\circ} \mathrm{C}$. Then $150 \mu \mathrm{l}$ of dimethyl sulfoxide (DMSO, Sigma-Aldrich, St. Louis, USA) was added to each well and thoroughly mixed for $10 \mathrm{~min}$. Spectrometric absorbance at 
a wavelength of $570 \mathrm{~nm}$ was determined with a microplate reader (model 680; Bio-Rad, Hercules, USA). Each sample had three replicates.

Cell cycle analysis. The cell cycle phase distribution was determined by flow cytometry. The cells were harvested $48 \mathrm{~h}$ after transfection (at an exponential growth phase) and single-cell suspensions containing $1 \times 10^{6}$ cells were fixed with $75 \%$ alcohol. The cell cycle was monitored using propidium iodide (PI) staining of the nuclei. The fluorescence of DNA-bound PI in the cells was measured with a FACScan flow cytometer (BD Pharmingen, San Diego, USA) and the results were analyzed with ModFit 3.0 software (Verity Software House, Topsham, ME). Proliferative index (PI) = $(\mathrm{S}+\mathrm{G} 2 / \mathrm{M}) /(\mathrm{G} 0 / \mathrm{G} 1+\mathrm{S}+\mathrm{G} 2 / \mathrm{M})$.

Cell invasion assay. Transwell invasion assays were performed using SGC-7901 cells cultured in 24-well $8 \mu \mathrm{m}$ pore size transwell plates according to the manufacturer's instructions (Corning, New York, USA). The bottom of transwell chamber was coated with 1:8 diluted BD Matrigel ${ }^{\mathrm{m}}$ Basement Membrane Matrix (final concentration $0.5 \mathrm{mg} / \mathrm{ml}$ ) and air-dried at $4^{\circ} \mathrm{C}$. The membranes were rehydrated with warm serum-free PRMI 1640 Media $(1.0 \mathrm{~mL} /$ chamber) for $2 \mathrm{~h}$. The upper chamber was filled with $1 \times 10^{5}$ cells in serum-free PRMI 1640 Media containing 5\% FBS. The lower chamber was filled with PRMI 1640 Media containing 25\% FBS as a chemo-attractant. After the chambers were incubated for $24 \mathrm{~h}$ at $37^{\circ} \mathrm{C}$ in an atmosphere containing $5 \% \mathrm{CO}_{2}$, non-invading cells on the upper side of the chamber were removed from the surface of the membrane by scrubbing, and invading cells on the lower surface of the membrane were with $100 \%$ methanol, mounted and dried at $80^{\circ} \mathrm{C}$ for $30 \mathrm{~min}$. The number of cells invading through the matrigel was counted by a technician blinded to the experimental settings in four randomly selected microscopic fields of each filter, using an inverted microscope at $200 \times$ magnification. Each test was repeated triplicately.

Tumor xenograft. Eighteen 3-4 weeks old BALB/c mice weighing 18-22 g were obtained from Department of Zoology, Central South University (Changsha, China). Mice were housed in microisolator cages with autoclaved bedding in a specific pathogen-free facility. Animals were observed for signs of tumor growth, activity, feeding and pain in accordance with the guidelines of the Research Ethics Committee on Animals, Central South University, China. Suspensions of tumor cells SGC-7901 ( $2 \times 10^{7}$ viable cells/mouse) were implanted into the axillary fossa region of BALB/c mice as previously described. When the tumors grew to $52.2 \pm 6.9$ $\mathrm{mm}^{3}$, the animals were randomly divided into three groups $(n=6)$ that received the following treatment groups: 1$)$ black control group, which received only $0.9 \%$ sodium chloride intratumorally twice a week; 2 ) negative control group, which received non-silencing siRNA + liposome intratumorally twice a week; and 3) EphA2 siRNA group, which received EphA2 siRNA + liposome intratumorally twice a week. The width and length of each tumor were measured once a week using a caliper, and the tumor volume was estimated by the following equation: $\mathrm{V}=$ width $^{2} \times$ length $\times \pi / 6$. Three weeks after treated with different means, the mice were sacrificed and the weight of the tumor was recorded. Tumor specimens were fixed in formalin overnight and embedded in paraffin for immunohistochemical examination.

Immunohistochemistry. Immunohistochemical staining was performed to detect the expression of EphA2 and MMP-9 in according to DAKO EnVision ${ }^{\mathrm{TM}}$ System (Dako, Glostrup, Denmark). Briefly, following a brief proteolytic digestion and a peroxidase blocking of tissue slides using $2.5 \%$ hydrogen peroxide in methanol for $30 \mathrm{~min}$ at room temperature, the slides were incubated with primary antibody against EphA2 (sc-924, dilution 1:100), and MMP-9 (sc-6840, dilution 1:200) (both from Santa Cruz, CA, USA) overnight at $4^{\circ} \mathrm{C}$, respectively. After washing, the slides were then incubated with peroxidase labeled polymer and substrate-chromogen. Finally, specimens were incubated in PBS containing diaminobenzidine (DAB) for $5 \mathrm{~min}$. An Olympus microscope was employed to visualize the staining of targeted proteins.

Statistical analysis. Quantitative data are expressed as the mean \pm standard deviation (SD). Statistical differences were evaluated by unpaired Student's t-test using statistical SPSS software (version 13.0, SPSS, Inc., Chicago, USA). A value of $P<0.05$ was considered statistically significant.

\section{Results}

RNA interference silences the EphA2 expression in SGC7901 cells. To examine the specific effect of EphA2 siRNA on EphA2 expression in the SGC-7901 cell line, the expression levels of EphA2 mRNA and protein were determined quantitatively using real-time RT-PCR and western blot analyses at $48 \mathrm{~h}$ post-transfection, respectively. Compared to cells transfected with non-silencing siRNA, the EphA2 mRNA level in cells transfected with EphA2 siRNA plasmid was reduced by $73.4 \%$ (Figure $1 \mathrm{~A}$ ). In agreement with the real-time RTPCR results, the EphA2 protein expression level was reduced by $65.1 \%$ in the SGC-7901 cells transfected with the EphA2 siRNA compared to the cells transfected with the non-silencing siRNA (Figure 1B, C). Therefore, these data demonstrate that EphA2 siRNA efficiently suppresses EphA2 expression at both mRNA and protein levels.

Silencing of EphA2 inhibits the proliferation of SGC7901 cells. To determine whether silencing of EphA2 affected the proliferation of SGC-7901 cells, cell growth was determined after 24, 48, 72 and 96 h using a MMT assay. Compared to the SGC-7901 cells transfected with the non-silencing siRNA, the viability of SGC-7901 cells was significantly decreased after EphA2 siRNA transfection at each of the different time point $(24,48,72$, and $96 \mathrm{~h})(P<0.01$, Figure 2). These proliferation curves indicate that the loss of EphA2 expression inhibits the proliferation of SGC-7901 cells in vitro.

Silencing of EphA2 delays the cell cycle in SGC-7901 cells. We determined the effect of EphA2 siRNA on the 
A

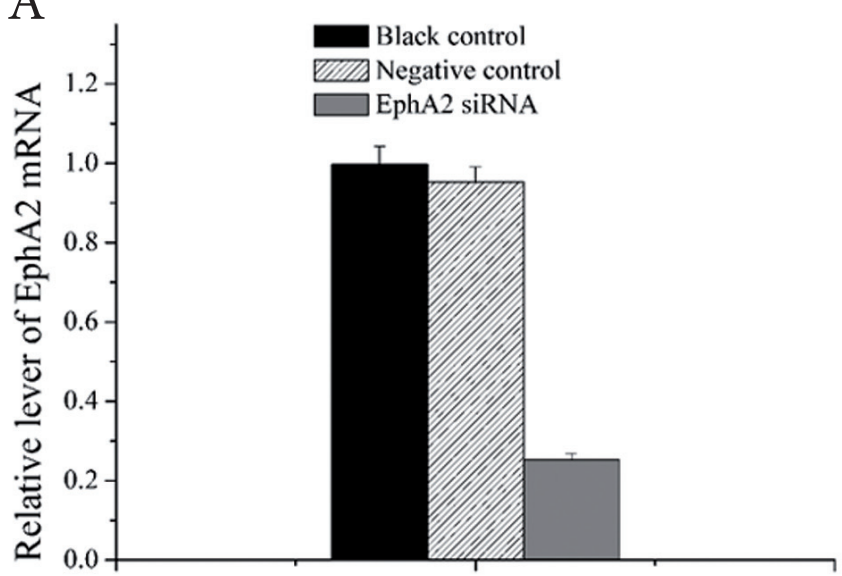

Experiment group

B

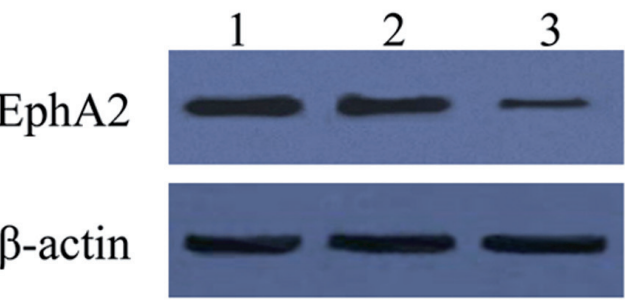

$130 \mathrm{kDa}$

C

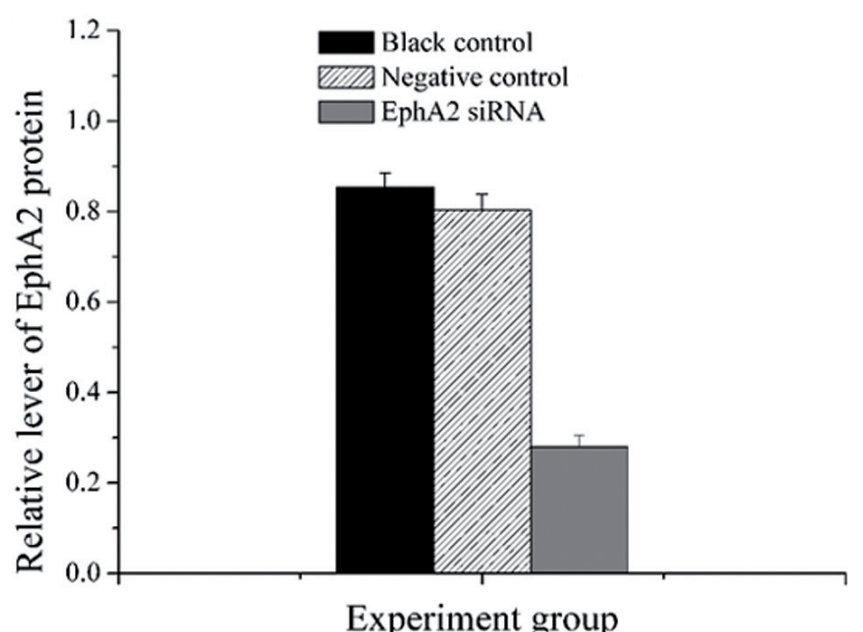

Figure 1. Silencing of EphA2 protein and mRNA expression in EphA2 siRNA treated SGC-7901 cells. A, The ratio of EphA2 to $\beta$-actin for mRNA levels. B, Level of EphA2 protein expression was determined by western blot analysis. Lane 1: black control group; Lane 2: negative control group; and Lane 3: EphA2 siRNA group. C, The ratio of EphA2 to $\beta$-actin for protein levels. Data are expressed as the mean \pm SD of three independent experiments. Compared to black and negative control group, both $\mathrm{mRNA}$ and protein levels of EphA2 were significant reduced in EphA2 siRNA group $(P<0.01)$.

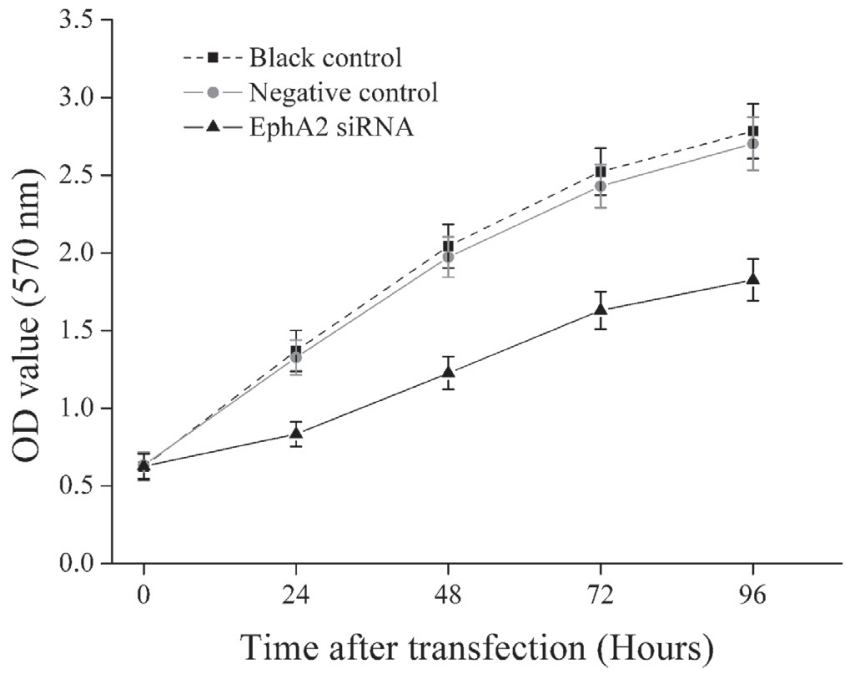

Figure 2. Silencing of EphA2 expression decreased cell proliferation in SGC-7901 cells. The data are represented as the mean \pm SD from triplicate assays and are representative of three independent experiments. After EphA2 siRNA transfection, the viability of SGC-7901 cells was evidently decreased at different time point $(24,48,72$, and $96 \mathrm{~h}$, respectively) $(P<0.01)$.

cell cycle of SGC-7901 cells by flow cytometry. Compared to black and negative control group, specific EphA2 siRNA increased the number of cells in the G0/G1 phase, decreased the number of cells of in the $S$ and $\mathrm{G} 2 / \mathrm{M}$ phase at $48 \mathrm{~h}$ after transfection $(P<0.05$, Table 1 , Figure 3$)$. It also showed that EphA2 siRNA treatment resulted in a dramatic low level of PI of SGC-7901 cells $(P<0.05$, Table 1$)$. These results show that down regulation of EphA2 expression delays SGC-7901 cell cycle progression in vitro.

Silencing of EphA2 suppresses the cell invasion of SGC7901 cells. To determine whether EphA2 plays an important role in the cell invasion of the SGC-7901 cells, we performed cell invasion assays using a transwell system. As shown in Figure 4, down-regulation of EphA2 by EphA2 siRNA inhibited the invasiveness of SGC-7901 cells compared with untransfected cells and non-silencing siRNA transfected cells, as indicated by a marked decrease in the number of cells that invaded the bottom well $(P<0.01)$. However, there was no significant difference in the cell invasion between black and negative control group $(P>0.05)$. These results display that suppressing of EphA2 expression inhibits SGC-7901 cell inva-

Table 1. Comparison of the cell cycle distributions in three different groups

\begin{tabular}{lcccc}
\hline \multirow{2}{*}{ Groups } & \multicolumn{4}{c}{ Cell cycle } \\
\cline { 2 - 5 } & G0/G1 (\%) & S (\%) & G2/M (\%) & PI \\
\hline Black control & $51.26 \pm 2.28$ & $35.42 \pm 1.56$ & $13.32 \pm 0.77$ & $0.49 \pm 0.02$ \\
Negative control & $52.96 \pm 2.12$ & $34.01 \pm 1.46$ & $13.03 \pm 0.67$ & $0.47 \pm 0.02$ \\
EphA2 siRNA & $75.35 \pm 1.77$ & $14.35 \pm 0.84$ & $10.30 \pm 0.93$ & $0.24 \pm 0.02$ \\
\hline
\end{tabular}

Data are expressed as mean \pm SD from three experiments. 


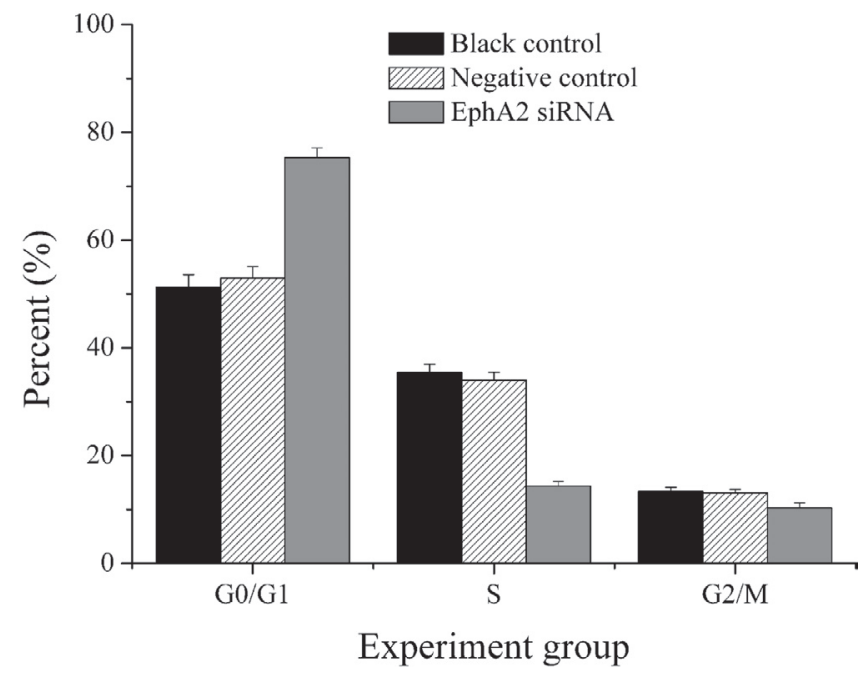

Figure 3. Silencing of EphA2 expression delayed cell cycle of SGC-7901 cells. The cell cycle stage was determined by flow cytometry, and each test was repeated three times. The data are expressed as the mean \pm SD of three independent determinations for each treatment group. Compared to black control and negative control group, SGC-7901 cells were blocked in the G0/G1 phase and sharply reduced in the G2/M, $\mathrm{S}$ phase by EphA2 siRNA $(P<0.05)$.

sion, which also indicated that EphA2 promoted cell invasion in SGC-7901 cells.

EphA2 siRNA decreases xenograft tumor growth of SGC-7901 cells. To investigate the potential effects of EphA2 siRNA on the inhibition of SGC-7901 cell growth in vivo, we injected either EphA2 siRNA with a liposome, non-silencing siRNA with a liposome, or single $0.9 \%$ sodium chloride into SGC-7901 tumor xenografts in nude mice. Both the volume and the weight of the tumors treated with the EphA2 siRNA were significantly decreased compared to those treated with the non-silencing siRNA or the $0.9 \%$ sodium chloride $(P<0.01$, Figure $5 \mathrm{~A}, \mathrm{~B})$. The rate of tumor inhibition in the EphA2 siRNA treated xenograft tumors weight was approximately $43.1 \%$. Compared to negative control group, black control group had no obvious differences in the volume or weight of the tumor xenografts. Hematoxylin and eosin (H\&E) stains show that all of the tumor tissues were gastric adenocarcinoma. Furthermore, we examined the expression of EphA2 in the tumor xenografts by immunohistochemistry. Moderate-to-strong immunoreactivity to EphA2 was observed in the cytoplasm of the carcinoma cells. The expres-

Figure 4. Silencing of EphA2 expression inhibited SGC-7901 cells invasion ability. A, Cells that had migrated into the lower chambers were counted under a microscope. Invasiveness of cells transfected with EphA2 siRNA was significantly reduced compared to that of cells transfected with negative control siRNA $(P<0.01)$. The data are expressed as the mean \pm SD of three independent determinations for each treatment group. B, Here shows one representative results of the triplicate experiments, black control group (left panel, 200x mag), the negative control group (middle panel, 200x mag), and the EphA2 siRNA group (right panel, 400x mag).

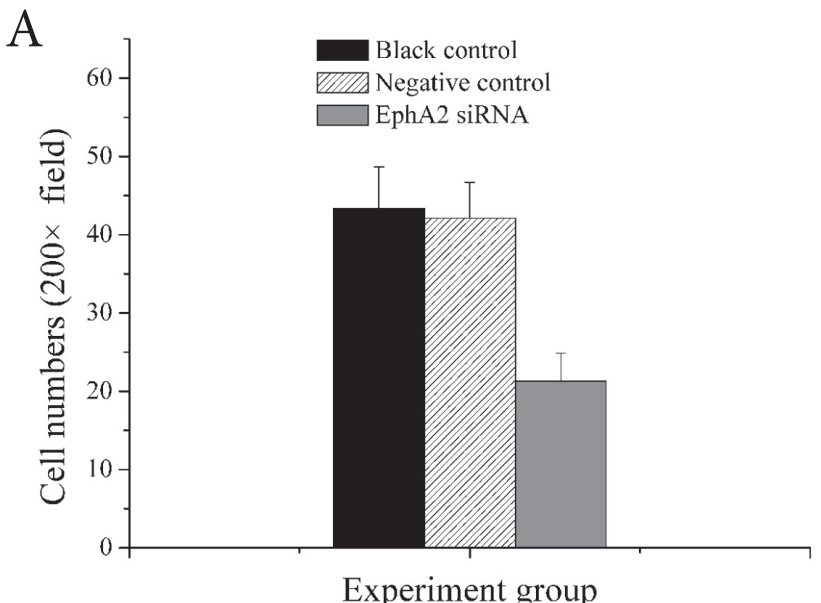

B
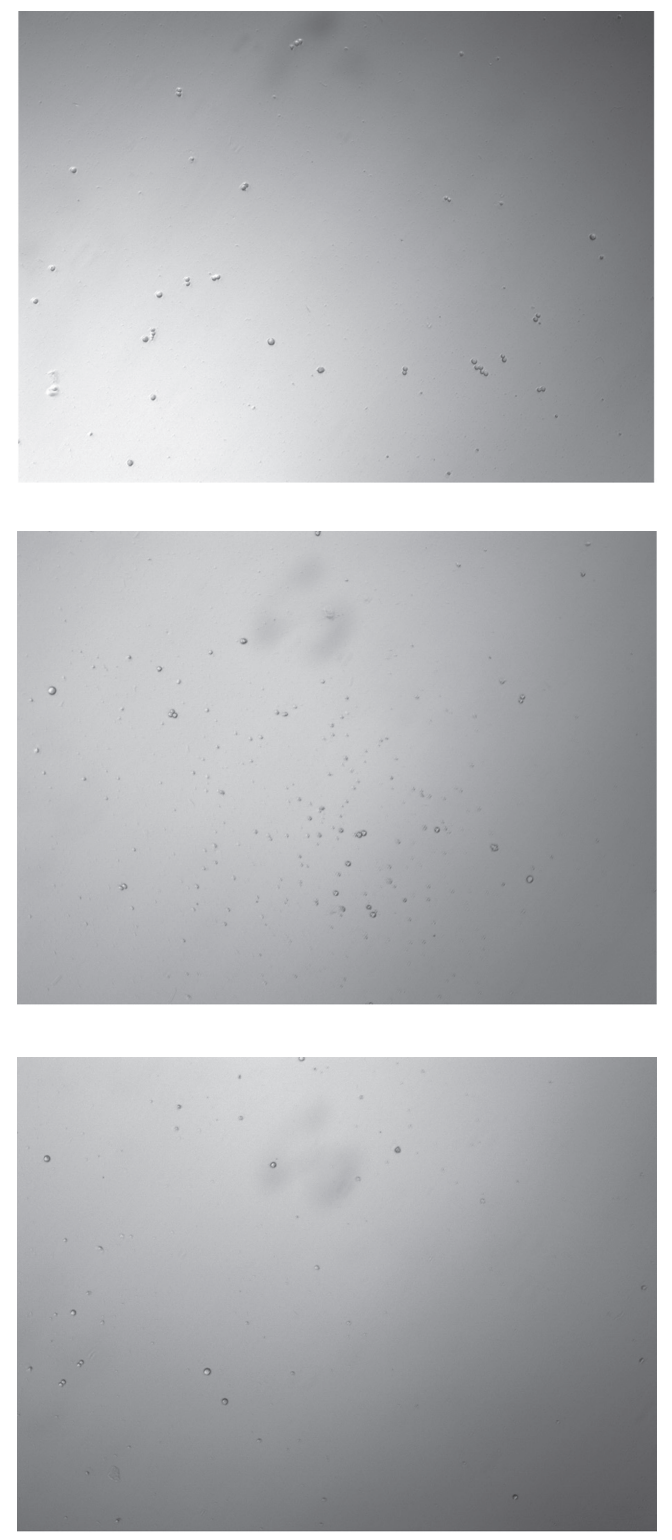
A

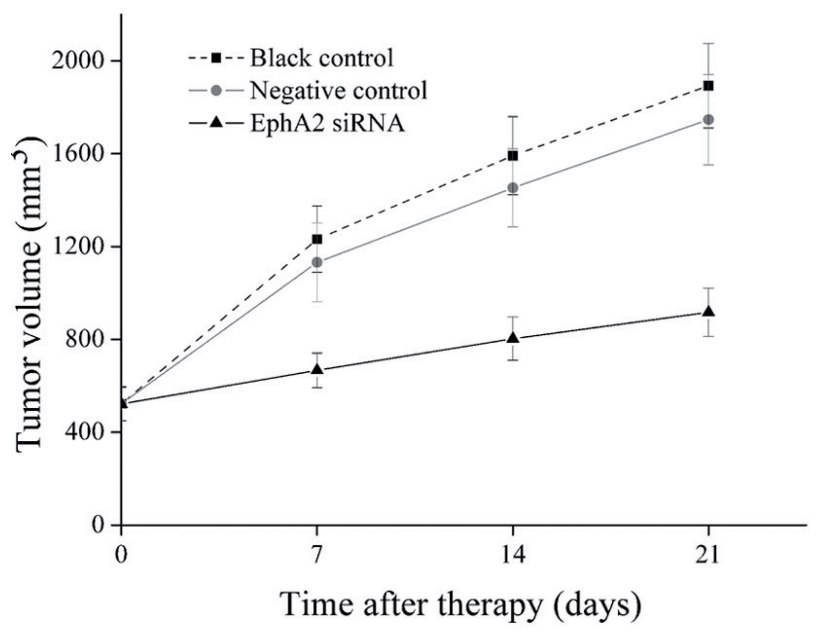

B

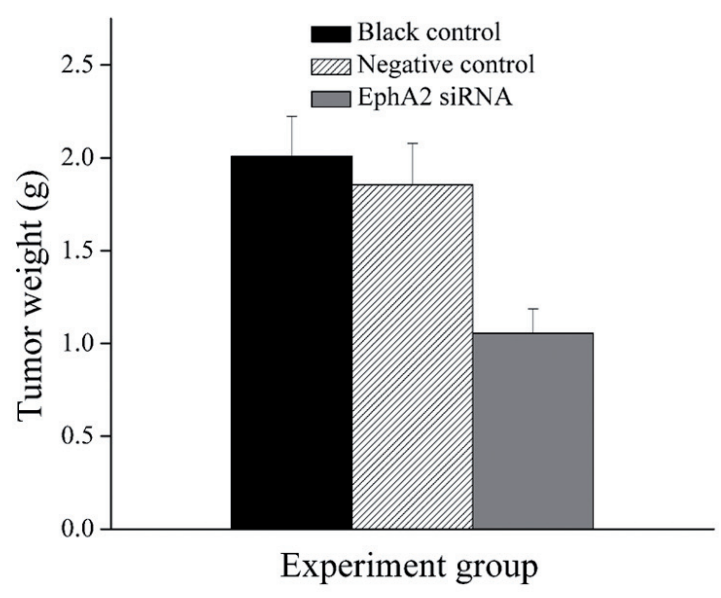

$\mathrm{C}$
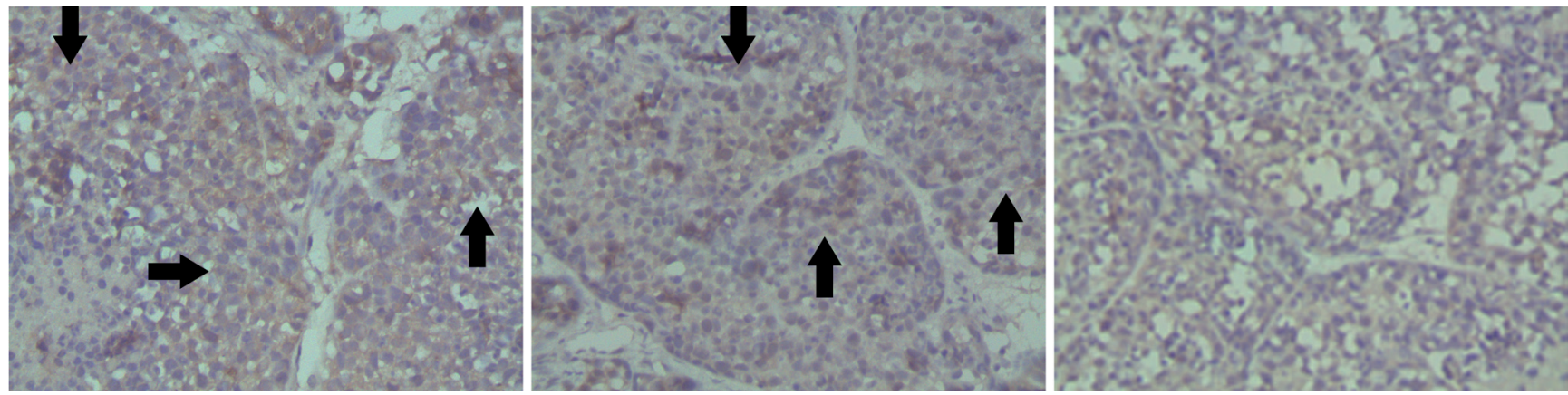

Figure 5. Intratumoral injection of EphA2 siRNA plasmid suppressed tumor growth and EphA2 expression in nude mice. Mice were injected subcutaneously in the axillary fossa with SGC-7901 cells $\left(2 \times 10^{7}\right.$ viable cells/mouse). Fourteen days after implantation, the mice were randomly divide into three groups $(n=6)$ and administered an intratumoral injection of either EphA2 siRNA, non-silencing siRNA, or only $0.9 \%$ sodium chloride. A, Tumor volume was measured at the indicated times and tumor growth curves are shown. The point indicates the mean tumor volume (calculated from six mice), while the bars indicate the SD. There was a statistically significant difference between the EphA2 siRNA group and either black or negative control group $(P<0.01)$. B, Tumors were removed 35 days after implantation and the tumor weights were measured. The data are expressed as the mean \pm SD from six mice. The decrease in the EphA2 siRNA group was significantly different compared to black or negative control group $(P<0.01)$. $C$, The expression of EphA2 was assayed by immunohistochemistry. The arrows indicate the positive staining. Strong expression of EphA2 in tissues from negative control group (left panel, 400x mag) and black control group (middle panel, 400x mag), moderate expression of EphA2 in tissues from EphA2 siRNA group (right panel, 400x mag).

sion of EphA2 was higher in the black and negative control group compared to the EphA2 siRNA group. Representative images of the immunohistochemistry staining of EphA2 are provided in Figure 5C.

Silencing of EphA2 suppresses the expression of MMP9 in SGC-7901 cells. In order to determine if silencing of EphA2 affected MMP-9 gene expression, we quantitated the expression of MMP-9 by real-time RT-PCR and western blot analysis at $48 \mathrm{~h}$ post-transfection in vitro (Figure $6 \mathrm{~A}, \mathrm{~B}, \mathrm{C}$ ). The expression of MMP-9 in tumor xenografts was also detected by immunohistochemistry in vivo (Figure 6D). Compared to untransfected cells and non-silencing siRNA transfected cells, the expression of MMP-9 mRNA and protein level in EphA2 siRNA transfected cells were decreased significantly $(P$
$<0.01)$. At the same time, the expression of MMP-9 protein was higher in $0.9 \%$ sodium chloride treated or non-silencing siRNA treated xenograft tumor tissues than this in EphA2 siRNA treated xenograft tumor tissues. Therefore, silencing of EphA2 expression can be accounted for the reduction of the MMP-9 expression in SGC-7901 cells.

\section{Discussion}

In the present study, we developed an efficient EphA2 siRNA plasmid to specifically knockdown EphA2 expression in gastric cancer SGC-7901 cells. Using this plasmid, silencing of the EphA2 expression level in SGC-7901 cells decreased cell proliferation and invasion ability in vitro. Intratumoral 
A

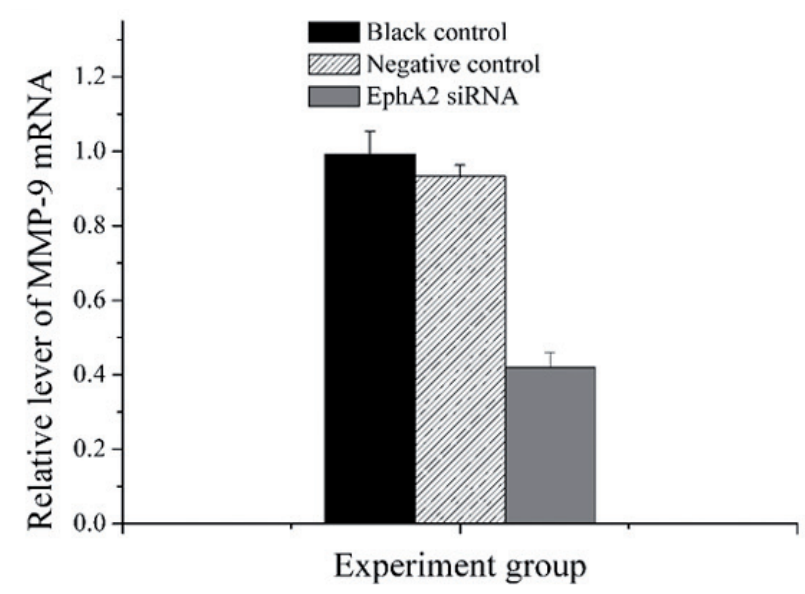

$\mathrm{C}$

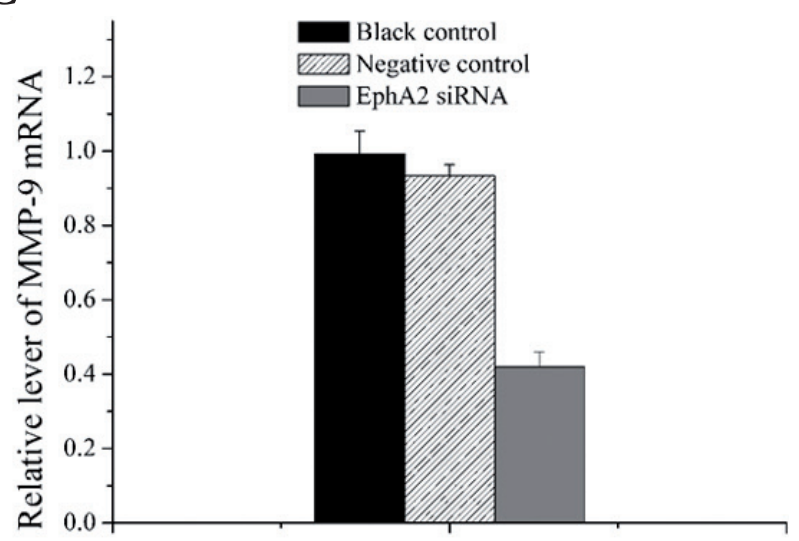

Experiment group

B

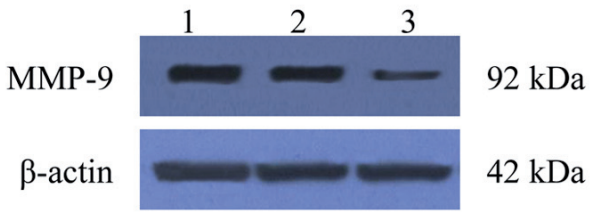

$\mathrm{D}$
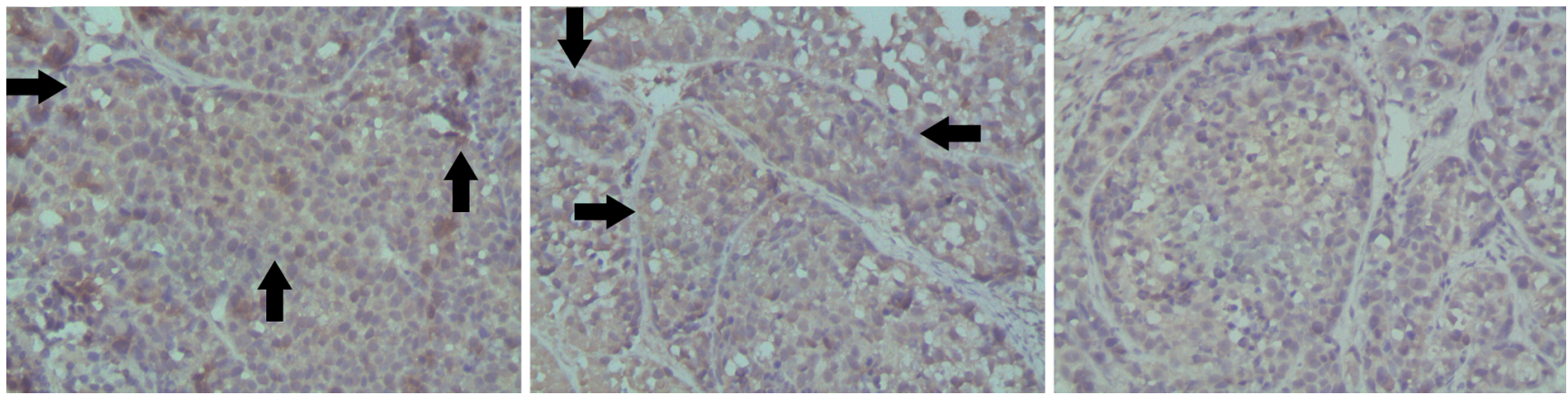

Figure 6. Silencing of EphA2 suppressesd expression of MMP-9 in in vitro and in vivo. A, The ratio of MMP-9 to $\beta$-actin for mRNA levels. B, Level of MMP-9 protein expression determined by Western blot analysis. C, The ratio of MMP-9 to $\beta$-actin for protein levels. Compared to black and negative control group, both mRNA and protein levels of MMP-9 were significant decreased in EphA2 siRNA group $(P<0.01)$. D, The expression of MMP-9 in xenograft tumors was assayed by immunohistochemistry. The arrows indicate the positive staining. Strong expression of MMP-9 in tissues from negative control group (left panel, 400x mag) and black control group (middle panel, 400x mag), moderate expression of MMP-9 in tissues from EphA2 siRNA group (right panel, 400x mag).

injection EphA2 siRNA plasmid also suppressed growth of SGC-7901 cells xenografts in vivo. Furthermore, decreasing the EphA2 expression level resulted in down-regulation of MMP-9 expression in vitro and in vivo.

EphA2 siRNA plasmid was constructed successfully and transfected into the SGC-7901 cells efficiently. Compared to negative control group, the mRNA and protein level of EphA2 in EphA2 siRNA group were reduced by $73.4 \%$ and $65.1 \%$, respectively. Using this specifically EphA2 siRNA, Duxbury et al have suppressed EphA2 protein expression by between 78 and $88 \%$ in pancreatic adenocarcinoma cells [16]. Meantime, Landen et al reported that EphA2 was highly effective in reducing in vivo by using the same EphA2 siRNA incorporated into the neutral liposome [17,18]. These data indicate that this EphA2 siRNA construct was effective and provided an appropriate system for the study of mitotic events under EphA2 knockdown conditions.

Our results demonstrate that the proliferation of gastric cancer cells was significantly inhibited upon loss of EphA2. EphA2 
siRNA resulted in an increase in the percentage of apoptotic and G0/G1 stage cells, as well as a significant reduction in the percentage of $S$ and G2/M stage cells. In addition, the EphA2 siRNA construct significantly decreased the invasion of the transfected SGC-7901 cells. Recent studies also suggested that silencing of EphA2 caused a significant reduction of tumor cells proliferation and invasion in vitro. Nasreen et al showed that silencing EphA2 expression inhibited proliferation, haptotaxis and induced apoptosis in malignant mesothelioma cells [19]. Zhou et al reported that the transfected EphA2 glioma cells showed lower proliferation, higher apoptosis, and loss of mitochondrial membrane potentiall [20]. Taddei et al's findings indicated that EphA2-overexpressing prostate carcinoma cells gained an invasive benefit from their amoeboid motility style to escape from primary tumors and then, enhancing their clonogenic potential successfully target bone and grow metastases [21]. The complexity of the EphA2 signalling has been nicely investigated in a study of Miao et al which demonstrated that EphA2 kinase has diametrically opposite roles in regulating chemotactic cell migration: ligand-independent promotion and ligand-dependent inhibition [22]. However, we only discussed EphA2 overexpression promoted migration without the impact of its ligand ephrin-A1 in this study.

To investigate the inhibitory role of EphA2 siRNA on the proliferation of gastric cancer cells in vivo, BALB/c-nu mice with SGC-7901 cell xenograft tumors were intratumoral injected with EphA2 siRNA plasmid. Our results demonstrate that both the volume and the weight of the tumors in EphA2 siRNA group were significantly decreased compared to black control group or negative control group. The rate of tumor inhibition in the EphA2 siRNA treated xenograft tumors weight was approximately $43.1 \%$. The expression of EphA2 protein was higher in the black and negative control group compared to the EphA2 siRNA group. Landen et al also reported that intraperitoneal injection of EphA2 siRNA incorporated in neutral liposome reduced orthotopic ovarian tumor growth by $48-81 \%$ compared to control siRNA [18]. Taken together, these data show that decreasing the expression of EphA2 by RNA interference significantly inhibited the proliferation and invasion of SGC-7901 gastric cancer cells in vitro and in vivo, which indicate that EphA2 play an important role in tumor proliferation and invasion.

Acquisition of cell migratory and invasive abilities is essential for cancer invasion and metastasis. One of the key steps in the cancer invasion and metastatic cascade involves the disruption of ECM and basement membranes, which permits tumor cells to access a distant site. MMPs have the capacity to degrade virtually every component of ECM, enhance invasiveness of tumor cells and facilitate angiogenesis [10]. Therefore, MMPs have been regarded as major critical molecules assisting tumor cells during invasion and metastasis. Type IV collagen is a critical component of the basement membrane architectural scaffolding. MMP-9 (gelatinase B, 92kDa gelatinase) can degrade type IV collagen as a primary substrate, assisting the metastatic cancerous cells to pass through the basement membrane. Therefore, MMP-9 is considerable to be the most important mediators of tumor migration and invasion among MMPs [14].

Lin et al observed that EphA2 overexpression in ovarian cancer cells was significantly associated with strong stromal and epithelial MMP-9 [23]. Our study also shown that immunostaining expression of EphA2 protein was postively correlated with that of MMP-9 in hepatocellular carcinoma tissue [15]. Interesting, our present study show that the expression of MMP-9 mRNA and protein level in EphA2 siRNA group were decreased significantly compared to black and negative control group in vitro and in vivo. It means silencing of EphA2 expression inhibits the expression of MMP-9 in SGC-7901 cells, which indicated silencing of EphA2 may inhibit invasion of human gastric cancer cells through regulation of MMP-9. Duxbury et al reported that EphA2 overexpression induced and sustained phosphorylation and kinase activity of FAK, one consequence of which was to increase expression of another gelatinase matrix metalloproteinase-2 (MMP-2) [24]. Recently, Chen et al reported curcumin inhibited the growth of engrafted melanoma VM channels through the down-regulation of the EphA2/PI3K/ MMPs signaling pathway [25]. EphA2 have been shown to affect tumor cell migration and invasion downstream signaling pathways including Ras-mitogen-activated protein kinase (MAPK), PI3K/AKT, and integrins/FAK/paxillin/Rho [26]. The activation of MMPs is also associated with different signaling systems such as AP-1 and ETS transcription factors, PKC, PI3K, cAMP, and so on [27]. However, the possible underlying mechanism of how EphA2 regulate MMP-9 expression is still unclear at present and merit further investigation.

In conclusion, our results showed that silencing of the EphA2 expression by specific siRNA inhibited the expression inhibited the proliferation and invasion in vitro and in vivo. Furthermore, silencing of EphA2 expression also suppressed the expression of MMP-9. This study provides clear evidence that EphA2 is functionally important in the proliferation and invasion of gastric cancer and may serve as a new target for gastric cancer therapy.

Acknowledgements: This study was supported by grants from National Natural Science Foundation of China (No. 81172297), Graduate Degree Thesis Innovation Foundation of Central South University (No. 2960-71131100013) and Natural Science Foundation, Health Department of Hunan Province, China (no. B2010-010).

\section{References}

[1] AMIT I, WIDES R, YARDEN Y Evolvable signaling networks of receptor tyrosine kinases: relevance of robustness to malignancy and to cancer therapy. Mol Syst Biol 2007; 3: 151. http://dx.doi.org/10.1038/msb4100195

[2] SULMAN EP, TANG XX, ALLEN C, BIEGEL JA, PLEASURE DE et al. a human EPH-related gene,maps to 1p36.1, a com- 
mon region of alteration in human cancers. Genomics 1997; 40: 371-374. http://dx.doi.org/10.1006/geno.1996.4569

[3] WANG LF, FOKAS E, BIEKER M, ROSE F, REXIN P et al. Increased expression of EphA2 correlates with adverse outcome in primary and recurrent glioblastoma multiforme patients. Oncol Rep 2008; 19: 151-156.

[4] BRANNAN JM, DONG W, PRUDKIN L, BEHRENS C, LOTAN R et al. Expression of the receptor tyrosine kinase EphA2 is increased in smokers and predicts poor survival in non-small cell lung cancer. Clin Cancer Res 2009; 15: 44234430. http://dx.doi.org/10.1158/1078-0432.CCR-09-0473

[5] CUI XD, LEE MJ, YU GR, KIM IH, YU HC et al. EFNA1 ligand and its receptor EphA2: potential biomarkers for hepatocellular carcinoma. Int J Cancer 2010; 126: 940-949. http://dx.doi.org/10.1002/ijc.24798

[6] BAETEN CI, HILLEN F, PAUWELS P, DE BRUINE AP, BAETEN CG Prognostic role of vasculogenic mimicry in colorectal cancer. Dis Colon Rectum 2009; 52: 2028-2035. http://dx.doi.org/10.1007/DCR.0b013e3181beb4ff

[7] MERRITT WM, KAMAT AA, HWANG JY, BOTTSFORDMILLER J, LU C et al. Clinical and biological impact of EphA2 overexpression and angiogenesis in endometrial cancer. Cancer Biol Ther 2011; 10: 1306-1314. http://dx.doi. org/10.4161/cbt.10.12.13582

[8] YUAN WJ, GE J, CHEN ZK, WU SB, SHEN H et al. Overexpression of EphA2 and EphrinA-1 in human gastric adenocarcinoma and its prognostic value for postoperative patients. Dig Dis Sci 2009; 54: 2410-2417. http://dx.doi. org/10.1007/s10620-008-0649-4

[9] LU C, SHAHZAD MM, WANG H, LANDEN CN, KIM SW et al. EphA2 overexpression promotes ovarian cancer growth. Cancer Biol Ther 2008; 7: 1098-1103. http://dx.doi. org/10.4161/cbt.7.7.6168

[10] ROY R, YANG J, MOSES MA Matrix metalloproteinases as novel biomarkers and potential therapeutic targets in human cancer. J Clin Oncol 2009; 27: 5287-5297. http://dx.doi. org/10.1200/JCO.2009.23.5556

[11] LINN R, DUPONT BR, KNIGHT CB, PLAETKE R, LEACH RJ Reassignment of the $92-\mathrm{kDa}$ type IV collagenase gene (CLG4B) to human chromosome 20. Cytogenet Cell Genet 1996; 72: 159-161. http://dx.doi.org/10.1159/000134175

[12] LEE LY, WU CM, WANG CC, YU JS, LIANG Y et al. Expression of matrix metalloproteinases MMP-2 and MMP-9 in gastric cancer and their relation to claudin-4 expression. Histol Histopathol 2008; 23: 515-521.

[13] GAO ZL, ZHANG C, DU GY, LU ZJ Clinical significance of changes in tumor markers, extracellular matrix, MMP-9 and VEGF in patients with gastric carcinoma. Hepatogastroenterology 2007; 54: 1591-1595.

[14] DERYUGINA EI, QUIGLEY JP Matrix metalloproteinases and tumor metastasis. Cancer Metastasis Rev 2006; 25: 9-34. http://dx.doi.org/10.1007/s10555-006-7886-9

[15] YANG P, YUAN W, HE J, WANG J, YU L et al. Overexpression of EphA2, MMP-9, and MVD-CD34 in hepatocellular carcinoma: Implications for tumor progression and prognosis. Hepatol Res 2009; 39: 1169-1177. http://dx.doi.org/10.1111/ j.1872-034X.2009.00563.x
[16] DUXBURY MS, ITO H, ZINNER MJ, ASHLEY SW, WHANG EE EphA2: a determinant of malignant cellular behavior and a potential therapeutic target in pancreatic adenocarcinoma. Oncogene 2004; 23: 1448-1456. http://dx.doi.org/10.1038/ sj.onc. 1207247

[17] LANDEN CN JR, CHAVEZ-REYES A, BUCANA C, SCHMANDT R, DEAVERS MT et al. Therapeutic EphA2 gene targeting in vivo using neutral liposomal small interfering RNA delivery. Cancer Res 2005; 65: 6910-6918. http://dx.doi. org/10.1158/0008-5472.CAN-05-0530

[18] LANDEN CN, MERRITT WM, MANGALA LS, SANGUINO AM, BUCANA C et al. Intraperitoneal delivery of liposomal siRNA for therapy of advanced ovarian cancer. Cancer Biol Ther 2006; 5: 1708-1713. http://dx.doi.org/10.4161/ cbt.5.12.3468

[19] NASREEN N, MOHAMMED KA, ANTONY VB Silencing the receptor EphA2 suppresses the growth and haptotaxis of malignant mesothelioma cells. Cancer 2006; 107: 2425-2435. http://dx.doi.org/10.1002/cncr.22254

[20] ZHOU Z, YUAN X, LI Z, TU H, LI D et al. RNA interference targeting EphA2 inhibits proliferation, induces apoptosis, and cooperates with cytotoxic drugs in human glioma cells. Surg Neurol 2008; 70: 562-569. http://dx.doi.org/10.1016/ j.surneu.2008.04.031

[21] TADDEI ML, PARRI M, ANGELUCCI A, BIANCHINI F, MARCONI C et al. EphA2 induces metastatic growth regulating amoeboid motility and clonogenic potential in prostate carcinoma cells. Mol Cancer Res 2011; 9: 149-160 http://dx.doi.org/10.1158/1541-7786.MCR-10-0298

[22] Miao H, LI DQ, MUKHERJEE A, GUO H, PETTY A et al. EphA2 mediates ligand-dependent inhibition and ligandindependent promotion of cell migration and invasion via a reciprocal regulatory loop with Akt. Cancer Cell 2009; 16: 9-20. http://dx.doi.org/10.1016/j.ccr.2009.04.009

[23] LIN YG, HAN LY, KAMAT AA, MERRITT WM, LANDEN $\mathrm{CN}$ et al. EphA2 overexpression is associated with angiogenesis in ovarian cancer. Cancer 2007; 109: 332-340. http://dx.doi.org/10.1002/cncr.22415

[24] DUXBURY MS, ITO H, ZINNER MJ, ASHLEY SW, WHANG EE Ligation of EphA2 by Ephrin A1-Fc inhibits pancreatic adenocarcinoma cellular invasiveness. Biochem Biophys Res Commun 2004; 320: 1096-1102. http://dx.doi.org/10.1016/ j.bbrc.2004.06.054

[25] CHEN LX, HE YJ, ZHAO SZ, WU JG, WANG JT et al. Inhibition of tumor growth and vasculogenic mimicry by curcumin through down-regulation of the EphA2/PI3K/MMP pathway in a murine choroidal melanoma model. Cancer Biol Ther 2011; 11: 229-235. http://dx.doi.org/10.4161/ cbt.11.2.13842

[26] WYKOSKY J, DEBINSKI W The EphA2 receptor and ephrinA1 ligand in solid tumors: function and therapeutic targeting. Mol Cancer Res 2008; 6: 1795-1806. http://dx.doi. org/10.1158/1541-7786.MCR-08-0244

[27] CHAKRABORTI S, MANDAL M, DAS S, MANDAL A, CHAKRABORTI T Regulation of matrix metalloproteinases: an overview. Mol Cell Biochem 2003; 253: 269-285. http:// dx.doi.org/10.1023/A:1026028303196 